\title{
Insomnia in clients with chronic, work-related musculoskeletal pain in a work recovery rehabilitation program
}

\author{
Katherine Harman ${ }^{\mathrm{a}, *}$, Eileen Keating ${ }^{\mathrm{b}}$, Susan Mayes ${ }^{\mathrm{b}}$, Jane Walsh ${ }^{\mathrm{b}}$ and Sally MacCallum ${ }^{\mathrm{b}}$ \\ ${ }^{\text {a }}$ School of Physiotherapy, Dalhousie University, Halifax, Canada \\ ${ }^{\mathrm{b}}$ Work Recovery Program, WorkSafeNB's Rehabilitation Centre, St. John, Canada
}

Received 16 January 2012

Accepted 1 February 2013

\begin{abstract}
.
BACKGROUND: There is a high prevalence of sleep disturbance with people experiencing chronic pain. Although multidisciplinary rehabilitation programs address many contributing factors for chronic pain, the impact of insomnia on clients is not often measured.

OBJECTIVE: Two studies were used to: first explore the experience of insomnia in a group of clients with chronic pain and then, in a group enrolled in a six-week work recovery rehabilitation program, compare measures of sleep disturbance at entry and upon its completion.

PARTICIPANTS: Sixteen clients participated in focus groups and 29 completed questionnaires; $46 \%$ were women and the average age was 43 years. They had a wide range of work-related musculoskeletal injuries and all had chronic pain.

METHODS: First two, semi-structured focus group interviews explored sleep disturbance. Then a different set of participants completed three sleep questionnaires before and after completing a rehabilitation program.

RESULTS: Focus group participants described sleep disturbance consistent with clinical insomnia and how it had a considerable impact on their lives. Completed questionnaires confirmed the presence of sleep disturbance at admission into a six-week rehabilitation program and at discharge, most measures were unchanged.

CONCLUSIONS: Although chronic pain rehabilitation generally includes interdisciplinary approaches, specific attention to insomnia is not part of this chronic pain rehabilitation program and therefore it is not surprising that there was no appreciable change by the end of the program. However, because sleep disturbance is prevalent in the chronic pain population and in this sample, and has such a strong impact on the individual's daytime functioning, effective interventions directed at sleep restriction and stimulus control should complement chronic pain rehabilitation programs.
\end{abstract}

Keywords: Sleep disturbance, daytime functioning, occupation

\section{Introduction}

It might be expected that people with chronic pain would gain relief from their pain during sleep because of the general decrease in sensory and motor respon-

\footnotetext{
* Corresponding author: Katherine Harman, 5869 University Ave., PO Box 15000, Halifax, NS B3H 4R2, Canada. Tel.: +1 902494 8820; Fax: +1 902494 1941; E-mail: k.harman@ dal.ca.
}

siveness during this state [1,2]. However, there is a high prevalence of insomnia among those with painrelated conditions [3-6]. On their own, each condition is associated with poor quality of life and is a challenge to treat effectively. For example, results of prolonged insomnia include impairment of cognitive performance, persistent fatigue and mood disturbance [7, 8] and chronic pain is linked with "severe emotional, physical, economic and social stresses" [9] When the 
two are experienced together, a complicated clinical picture can include additional, significant psychological challenges such as fear [10,11], depression [12] and anxiety [13]. There is a growing literature regarding the putative interaction between these two clinically complex conditions.

Although there is yet much to be understood, the interaction of sleep and pain has been noted in clinical symptomatology and epidemiology as well as neurophysiological mechanisms. Observations of this phenomenon have led some to conclude that one condition causes an increased severity of the other [14,15]. An exploration of the sleep/pain relationship using selfreport and actigraphic measures (data derived from activity instruments that can proxy for a polysomnographic measure of sleep) found a predictive relationship among the variables [16]. A longitudinal study using clients with major burn injury found a bidirectional relationship with sleep onset insomnia at hospital discharge predicting long term pain, and increased pain severity at discharge predicting long-term sleep onset insomnia [17]. Research into the interaction of the two has extended into the laboratory setting, providing further support for the synergistic effects. For example, with experimental sleep restriction, normal, pain free adults will develop increased bodily pain [18], and when the previous night's sleep was less than six hours, the reported next day pain levels increased [19].

Neurophysiologic mechanisms also provide some insight into the sleep/pain relationship. Sensory processing of pain signals use multiple pathways and also have been shown to be subject to considerable neuroplastic change such that chronic pain is conceptualized as a central, rather than a peripheral phenomenon [20]. Along with this shift in neurophysiologic processing, there is a change in the meaning of pain as it becomes associated with certain activities as well as feelings of fear and failure [21]. These processes are occurring in the presence of the powerful drive that the experience of pain has on our system, making it extremely difficult to ignore [22]. Sleep is regulated through the ascending arousal system which has a pathway through the thalamus to cortical targets [23]. In the sleep state, reduced thalamocortical activation is associated with a decrease in sensorimotor transmission (this is heralded by increased sigma power on EEG recordings) [24,25]. The finding of a decrease in sigma power in clients with chronic low back pain has been interpreted as increased sensory processing during sleep [26]. In the presence of insomnia, increased activity of corticolimbic sites, as measured by Positron Emission Tomogra- phy (PET), suggests a hyperaroused state [27]. And, multiple arousals is a feature of sleep in chronic pain conditions [28-30]. It is unknown if the sensitized nervous system contributes to hyperarousal and increased sensory processing, but it is likely that these central nervous system changes contribute to the prevalence of sleep disturbance in chronic pain. These findings reveal a compelling dynamic interplay which should be considered when delivering therapies, in particular, for chronic pain conditions.

With both chronic pain and insomnia, persistence of symptoms leads to maladaptive behaviours and beliefs, which in turn are believed to promote chronicity $[31,32]$. It is these maladaptive behaviours and beliefs that are the target of cognitive-behavioural (CBT) approaches in rehabilitation [33-35]. Multidisciplinary chronic pain programs generally include CBT to provide the individual with skills that will assist them in pain self-management [36-38].

Cognitive Behavioural Therapy interventions for insomnia have been shown to be effective in several experiments conducted independent of pain rehabilitation programs [31,39-41]. These studies examined the short term impact of CBT interventions on insomnia symptoms of people with chronic pain. Each study revealed a positive impact on sleep disturbance using self-report measures, in the absence of significant change in pain scores. In regards to treatment for insomnia, there are three 'levels' of insomnia management: sleep hygiene, stimulus control and sleep restriction. This last step requires several weeks of coaching to assist an individual through the process. Individualised counselling is often added to address dysfunctional beliefs about sleep that might contribute to sleep onset difficulties [42,43]. The cognitive-behavioural approach to insomnia has value and studies have demonstrated that it helps provide clients with the tools they need to manage their symptoms, despite the use of CBT approaches. Although insomnia is a well-known clinical problem with clients with chronic pain, the impact of insomnia has only been explored in a limited way and often it is not comprehensively addressed in chronic pain rehabilitation programs.

Participants in this study were enrolled in a Work Recovery Program that used a multidisciplinary approach to provide work hardening or special protocols with a focus on the safe return of the participant to work. Various treatment components were used to address the physical, functional and psychosocial barriers to rehabilitation and/or return-to-work. Intensive therapy is designed to promote healing, increase strength, 
improve conditioning, reduce pain and restore function. Individual and group education included sessions on sleep disturbance and strategies to address sleep issues. Although client feedback indicated a $90 \%$ satisfaction rating with the rehabilitation provided to address sleep disturbance, we sought a better understanding of the experience of insomnia in these clients and their measurable response to services provided. To understand the scope of insomnia in rehabilitation clients with chronic pain as well as changes in sleep disturbance in clients undergoing a six week work recovery program, two separate studies were conducted with clients with clinical insomnia: focus group interviews were used to learn more about the primary sleep complaints of chronic pain rehabilitation clients and once completed, a second, independent study was undertaken to document baseline measures of sleep disturbance prior to entry into a six week work recovery program and upon its completion.

\section{Methodology/participants}

This study was reviewed and approved by the University Ethics Review Board as well as the Professional Practice Committee of the rehabilitation centre. Participants for both studies were recruited through the Work Recovery Program in a Rehabilitation Hospital. The Work Recovery Program included only a small amount of content regarding insomnia: one group session on sleep hygiene and individual discussions about sleep postures. Signed informed consent was obtained prior to participation in either study. The focus group participants were recruited through a mailing to all injured workers with chronic pain being treated at the time the focus groups were being scheduled. All those interested in participating returned two questionnaires to assess inclusion/exclusion criteria (see below). The first sixteen names of people randomly drawn that met the inclusion criteria formed the two focus groups. They were contacted and confirmed to be interested. Participants were included if their Insomnia Severity Index was greater than 8 (interpreted as clinical insomnia [44]) and if they indicated no known medical cause of insomnia (such as respiratory or neurological conditions).

Two, semi-structured focus group interviews were conducted on the hospital site in a meeting room. They were led by the principal investigator and a staff member at the hospital (but not part of the Work Recovery Program) was the recorder. The facilitator asked openended questions, with the following central questions in mind:
1. Can you describe the nature of your sleep problem?

2. Can you describe/tell me how you feel about it and how it is affecting your life?

3. What have you tried to improve your sleep?

The focus groups were recorded and transcribed.

After the completion of the focus groups, a second study was conducted to explore the stability of insomnia complaints of this population over the period of the work recovery program.

Recruitment for the questionnaire study occurred during the routine admission process for the Work Recovery Program. Once informed of the study, if they met the same inclusion criteria (above), they were invited to participate. All participants completed a standardized, intake battery of questionnaires and assessments by a team of health professionals and were admitted into a six-week, work recovery rehabilitation program (the data from the comprehensive intake assessment are not presented in this paper). For this study, each participant completed three self-report questionnaires:

The Pittsburgh Sleep Quality Index (PSQI) [45], a one-month retrospective self-report questionnaire for sleep quality. The PSQI is a 10-item questionnaire that records sleep habits (e.g., time to bed, sleep onset, actual sleep time) and frequency of types of sleep disturbance (e.g., snoring, feeling hot, having pain) during the previous month, as well as reports of sleep quality and daytime sleepiness. Internal homogeneity, consistency and validity testing were reported as acceptable and clinical properties suggested the questionnaire could be used in clinical and research practice [45].

The Insomnia Severity Index (ISI), is a fifteen-item questionnaire with a rating scale of 5 points for statements about difficulty falling asleep and staying asleep as well as satisfaction of sleep pattern and being worried about the current sleep problem. It was designed for both clinical and research use to quantify perceived insomnia severity. Psychometric property testing showed that the ISI has adequate internal consistency and is reliable to measure perceived sleep difficulties. Further study revealed the instrument as a valid and sensitive measure and is used to determine the effectiveness of insomnia treatment $[44,46]$.

The Dysfunctional Beliefs and Attitudes about Sleep $-10(D B A S)$ [47], is a 10-item questionnaire with items that reflect the individual's beliefs about sleep (including causes for insomnia, sleep expectations, control and predictability of sleep and consequences of insomnia) $[33,48]$. This instrument was reduced to ten 
items through multiple studies that revealed three factors: Factor 1 (five items) - beliefs about the immediate negative consequences of insomnia, Factor 2 (three items) - beliefs about the long-term negative consequences of insomnia and Factor 3 - beliefs about the need for control over insomnia[48].

\section{Results}

\subsection{Study 1}

Two, two hour focus groups were completed; each with eight participants (average age $=41$ yrs. $50 \%$ were women), with various work-related injuries resulting in chronic pain and clinical insomnia. Transcripts were coded and analysed for themes through constant comparison. The findings are summarized below with quotes from the participants to illustrate the theme.

The description of the participants' sleep disturbance was consistent with insomnia. Participants described their sleep as unrefreshing, with significant difficulty at sleep onset and waking after sleep onset and having trouble falling asleep again. They described being extremely light sleepers; the slightest noise would wake them up. This insomnia was described as highly distressing with an impact on every aspect of their lives, including their pain condition. "The pain-sleep problem is a vicious cycle."

To address this insomnia, participants used prescription and over-the-counter medication as well as nonpharmacologic interventions for their sleep and pain problems. They reported using anti-inflammatories, opioids, anti-convulsants, anti-depressants, as well as alcohol, marijuana and other street drugs. They found some physical relief with baths, pillows and positioning for comfort, a new mattress, heating pad, TENS machine, moist heat, breathing techniques, outdoor exercise, relaxation tapes and distraction with hobbies, $\mathrm{TV}$, crosswords, reading, car drives and pets.

The reported sleep disturbance had a high impact on the participants' lives and they reported that they were preoccupied by their sleep and pain problems. They described having the constant (day and night) worry about not having enough sleep. "Worrying about sleep is an all day, all-consuming thing. It starts right at the beginning of the morning." "Some nights, I am petrified of going to bed."

The participants attributed their insomnia to the psychological stress in their lives, such as the uncertainty of their future and racing thoughts, as well as the physical stress of the pain, such as having difficulty finding a comfortable position or being awakened from sleep with the feeling that they had been asleep for only a very brief time. "The pain starts with muscle spasms when I am about to doze off, then the ache starts."

In addition to having their sleep disturbed by their thoughts and the experience of physical pain, participants reported that their dreams disrupted their sleep. Some had disturbing, scary dreams that they associated with their pain experience. "I dreamt that I was in a cage and it was being lowered into the water" this participant compared the dream to the feelings of helplessness of being handled by the insurance company. Others reported dream/sleep experiences consistent with sleep paralysis: "Nightmares. I can't move. I am awake but feel I cannot move." "I am screaming in my head." "I feel like someone is sitting on my chest." "I dream that I am drowning. I am trying to get up, but someone is holding me down."

Every participant agreed that the sleep disturbance had a considerable impact on their daytime functioning. The participants described a strong pressure to sleep during the day due to lack of sleep the night before and/or medication side effects. Participants reported feeling awful - tired and numb - most of the time; "like a zombie". Many of the medications they used "make me drowsy during the day", and the ones used for sleep had a 'hangover' effect. "I took sleeping pills, but felt groggy in the morning." The pressure to sleep led many to take naps, which then resulted in difficulty falling asleep at night. "I sleep all day off and on, on the couch." Associated with the fatigue and effects of medications, are reports of not being able to concentrate or remember things: "I used to read so much, now, I cannot keep focused enough to read even a simple book" "There is at least one time a day I feel like a complete idiot (due to memory loss)." "I can't remember who people are... it's embarrassing, a degrading experience."

The emotional impact of the sleep and pain problem is also significant. Participants reported changes in their relationships with other people resulting in social isolation. "I am just there.... I feel like a stranger in my house." This interferes with fun and intimacy. They reported being irritable, negative and angry, resulting in upset family relations. "My husband changed to working night shifts so he could get some sleep... the marriage has gone downhill." Participants reported being too tired and irritable to be social, preferring to be alone. "I don't want to talk (to people) anymore ... I 
don't have the patience anymore because I am so tired." "Someone tells me a joke, I take it personally." "I don't want to go out of my house anymore."

And, participants explained that all of this has a considerable impact on their feelings of self-worth. They felt very negative towards their future. They spoke of their deep disappointment of not being able to do what they used to do, wanting to give up and how difficult it is to go on each day. Hopelessness and helplessness make their life almost unbearable. Some spoke of thoughts of suicide and of other people they knew with chronic pain who had committed suicide.

\subsection{Study 2}

A complete set of pre/post questionnaires was collected from 29 participants, $46 \%$ were women and the average age was 43 years old. The participants had a wide range of work-related musculoskeletal injuries and all had a diagnosis of chronic pain. See Table 1 for questionnaire results.

Comparisons were made using a paired t-test $(\alpha<$ 0.05). For the Dysfunctional Beliefs and Attitudes questionnaire scores, each factor was compared for Pre (before the beginning of the rehabilitation program) and Post (after discharge). There was a statistically significant improvement in scores for the beliefs about the immediate negative consequences of insomnia (Factor 1). The two other factors, beliefs about: long term negative consequences and the need for control over insomnia, were not significantly different at the end of the program as compared to the beginning. Mean scores on the Pittsburgh Sleep Quality Index (11.9 and 11.4) did not change significantly and indicate moderately impaired sleep quality. The mean Insomnia Severity Index scores were not significantly different pre/post and are consistent with moderately severe clinical insomnia [44,46].

\section{Discussion}

This study examined the experience of insomnia in a sample of chronic pain clients in a rehabilitation program. Insomnia was explored through focus group interviews and self-report questionnaires. The findings revealed the presence of moderately severe clinical insomnia. The participants reported that insomnia affected their lives in many ways and for those undergoing a six-week work recovery program, their scores on self-report measures of sleep disturbance remained mostly unchanged.

The focus group participants' reports of their sleep disturbance clearly illustrated the psychological and physical stress of co-morbid sleep impairment and chronic pain. And, the fact that these stressors are entering their dream states reflects the deep-seated problem. Participants described experiences consistent with sleep paralysis (i.e., being conscious of surroundings but unable to move or speak and experiencing the feeling of pressure on their chest $[49,50])$. Sleep paralysis with hallucinations has been associated with sleep onset REM [50], which most often occurs in sleep deprived states as well as in the presence of depression [51].

The circadian effect of sleep disturbance was described by the participants. They were distressed over how their impaired sleep affected their daytime performance. Statements such as feeling "like a zombie", being embarrassed, irritable, angry and in despair about their marriage, described the social impact of their situation. Not only did they report feeling disinterested in participating in social activities, but their family support structure appeared to be failing. These descriptions resonate with other reports such as a populationbased telephone survey that revealed significant life interference from pain, with $49 \%$ reporting insomnia, $36 \%$ depression and $32 \%$ unable to work [52]. In addition, the focus group interviews provided evidence that participants engaged in dysfunctional daytime practices. For example, the experience of chronic pain often will lead individuals to lie down and rest during the day to manage their pain. However, naps, long periods recumbent and sleeping on and off have negative effects on night time sleep efficiency $[53,54]$. These habits are amenable to cognitive behavioural interventions such as stimulus control and sleep restriction, which have been demonstrated to be effective at improving the sleep efficiency of clients with chronic pain [55].

The strongest language from the participants came when they spoke about how the combination of chronic pain and insomnia made them feel miserable, isolated and hopeless. Chronic pain and insomnia independently rank highly as risk factors for suicidal ideation and suicide attempts [56-59]. An epidemiologic study of a large community-based sample found a unique and positive association between suicidal ideation and suicide attempts and one or more chronic pain conditions [58]. A more recent study of adolescents reported a similar relationship [59]. In addition, this study suggested that the presence of insomnia could contribute 
Table 1

Results from three questionnaires

\begin{tabular}{lrrcc}
\hline Measures $(n=29)$ & Mean score & SD & Paired t (Pre vs. Post) & p-value (Pre vs. Post) \\
\hline DBAS & & & & \\
Pre-F1 & 23.8 & 5.6 & 3.198 & 0.005 \\
Post-F1 & 20.8 & 4.8 & & 0.129 \\
Pre-F2 & 14.4 & 3.3 & 1.677 & 0.406 \\
Post-F2 & 13.0 & 4.1 & & \\
Pre-F3 & 9.7 & 2.7 & 1.335 & 0.135 \\
Post-F3 & 8.7 & 3.2 & & \\
ISI & 17.3 & 5.6 & 1.51 & 0.635 \\
Pre & 15.9 & & & \\
Post & & & & \\
PSQI & 11.9 & 4.5 & 0.48 & \\
Pre & 11.4 & 5.2 & & \\
Post & & & &
\end{tabular}

to the positive association found. These two chronic conditions take a serious toll on an individual.

In regards to the questionnaire data, all three measures confirmed the presence of sleep disturbance at admission and all but one (Factor 1 DBAS) were unchanged following a six-week rehabilitation program. Perhaps this finding is not surprising because this program had minimal content to address insomnia in their clients (only one session on sleep hygiene and individual consultation about sleep postures). The sample was similar to patients in other rehabilitation settings - they all had work-related chronic pain and were of working age (23-57 yrs.). This study revealed that the clients had moderately high scores on three measures of sleep disturbance.

Excessive focus and worry over sleep loss has been demonstrated to contribute to the perpetuation of insomnia [33]. Poor sleepers have been found to hold more dysfunctional beliefs and attitudes than good sleepers [60]. It has been suggested that CBT be used to treat insomnia through changing dysfunctional beliefs and attitudes about sleep because of the demonstrated relationship between attitudes and beliefs and measurable sleep outcomes (polysomnography and sleep diaries) [33]. Not only are treatment options available to improve their quality of life, it is important to consider the impact of insomnia on clients' ability to participate in rehabilitation programs and ultimately return to work. The focus groups revealed thoughts of helplessness and suicide. Patients should be carefully screened for the potential of self harm. They may also have difficulty learning new things, have interfering thoughts that could distract them from their program and they may also lack the motivation to succeed given the reciprocal impact of sleep disturbance on daytime functioning. Effective interventions should be sought out and incorporated into chronic pain rehabilitation programs.
The multidisciplinary rehabilitation team approach is widely used for chronic pain management, and provides the opportunity to address the multi-faceted problems of an individual with chronic pain. Different professional groups are represented within these multidisciplinary teams depending on the clientele, personnel and resources available. Each team member brings their professional expertise to the design of the rehabilitation program that often has a generic core program with additional individualized aspects for client-specific issues. Cognitive behavioural and selfmanagement approaches can be applied to address insomnia associated with chronic pain $[31,40]$. An eightweek study showed that CBT could facilitate change in sleep behaviours in community dwelling individuals in chronic pain with co-morbid insomnia [61]. The addition of CBT interventions for insomnia into chronic pain rehabilitation programs makes sense on many levels.

\section{Conclusion}

The evolution of care for chronic pain has moved towards interdisciplinary approaches, and when possible, earlier intervention. There is strong evidence that a large percentage of clients with chronic pain experience insomnia. In the group of injured workers who participated in the work recovery rehabilitation program and who reported moderate levels of insomnia, this study found improvement on only one measure (F1 reflecting the workers' beliefs about the immediate negative consequences of insomnia), with otherwise no change. The results of this study suggest that insomnia in clients with chronic pain should be directly addressed. Because sleep disturbance is prevalent and has such a strong impact on the individual's daytime func- 
tioning, it would appear that interventions directed at sleep restriction and stimulus control should complement chronic pain rehabilitation programs to impact the cyclicity of the complex interactions among sleep and chronic pain.

\section{Acknowledgements}

This study was supported by funding from WorkSafeNB. We appreciate the participation of the patients in this study.

\section{References}

[1] Steriade M. Thalamic Oscillations and Signalling. New York: Wiley, 1990.

[2] Monk TH. Sleep, Sleepiness and Performance. New York: John Wiley and Sons, 1991.

[3] Haythornthwaite J, Hegel MT, Kerns RD. Development of a sleep diary for chronic pain patients. J Pain and Symptom Manage 1991; 6: 65-72.

[4] Moffitt PF, Kalucy EC, Kalucy RS, Baum FE, Cooke RD. Sleep difficulties, pain and other correlates. J Int Med 1991; 230: $245-9$.

[5] Smith M, Haythornthwaite J. How do sleep disturance and chronic pain inter-relate? Insights from the longitudinal and cognitive-behavioural clinical trials literature. Sleep Med Rev 2004; 8: 119-32.

[6] Wooten V. Medical causes of insomnia. In: Kryger MH, Roth T, Dement WC, editors. Principles and Practice of Sleep Medicine.Philadelphia: W.B. Saunders Co.; 1989. p. 456-75.

[7] Carskadon M, Dement W. Normal human sleep: An overview. In: Kryger MH, Roth T, Dement WC, editors. Principles and Practice of Sleep Medicine.Philadelphia: W.B. Saunders Company, 1989, p. 3-13.

[8] Chugh D, Weaver T, Dinges D. Neurobehavioural consequences of arousals. Sleep 1996; 19(10): S198-S201.

[9] Bonica J. Importance of the Problem. In: Aronoff G, editor. Evaluation and treatment of chronic pain. 2nd ed. Baltimore: Williams and Wilkins; 1992. p. xx-xxviii.

[10] Dijkstra A, Vlaeyen J, Rijnen H, Nielson W. Readiness to adopt the self-management approach to cope with chronic pain in fibromyalgic patients. Pain 2001; 90(1-2): 37-45.

[11] Vlaeyen J, Linton S. Fear-avoidance and its consequences in chronic musculoskeletal pain: a state of the art. Pain 2000; 85: 317-32.

[12] Wilson K, Eriksson M, D’Eon JL, Mikail S, Emery P. Major depression and insomnia in chronic pain. Clin J Pain 2002; 18: $77-83$.

[13] McCracken L, Gross RT. Does anxiety affect coping with chronic pain? Clin J Pain 1993; 9: 253-9.

[14] Kundermann B, Krieg J-C, Schrieber W, Lautenbacher S. The effect of sleep deprivation on pain. Pain Res Manage 2004; 9(1): 25-32.

[15] Lautenbacher S, Kundermann B, Krieg J-C. Sleep deprivation and pain perception. Sleep Med Rev 2006; 10: 357-69.

[16] Chung K-F, Tso K-C. Relationship between insomnia and pain in major depressive disorder: A sleep diary and actigraphy study. Sleep Medicine 2010; 11: 752-8.
[17] Smith M, Klick B, Kozachik S, Edwards R, Holavanahalli $\mathrm{R}$, Wiechman S, et al. Sleep onset insomnia symptoms during hospitalization for major burn injury predict chronic pain. Pain 2008 Sep 15; 138(3): 497-506.

[18] Haack M, Mullington J. Sustained sleep restriction reduces emotional and physical well-being. Pain 2005; 119: 56-64.

[19] Edwards R, Almeida D, Klick B, Haythornthwaite J, Smith M. Duration of sleep contributes to next-day pain report in the general population. Pain 2008 Jun 30; 137(1): 202-7.

[20] Woolf C. Central sensitization: Implications for the diagnosis and treatment of pain. Pain 2010; In Press, Corrected Proof.

[21] Vlaeyen J. Fear in Musculoskeletal Pain. In: Dostrovsky JO, Carr D, Koltzenburg M, editors. Progress in Pain Research and Management.Seattle: IASP Press; 2003. p. 631-50.

[22] Eccleston C, Crombez G. Pain demands attention: A cognitive-affective model of the interruptive function of pain. Psychol Bull 1999; 125(3): 356-66.

[23] Saper C, Scammell T, Lu J. Hypothalamic regulation of sleep and circadian rhythms. Nature 2005 Oct 27; 437(7063): 125763.

[24] Steriade M, Domich L, Oakson G, Deschenes M. The deafferented reticular thalamic nucleus generates spindle rhythmicity. J Neurophysiol 1987; 17: 105-17.

[25] Steriade M. Basic mechanisms of sleep generation. Neurology 1992; 42(56): 9-18.

[26] Harman K, Pivik RT, D'Eon J, Wilson K, Swenson J, Matsunaga L. Sleep in depressed and nondepressed participants with chronic low back pain: electroencephalographic and behavioural findings. Sleep 2002; 25(7): 775-83.

[27] Nofzinger E, Buysse D, Germain A, Price J, Miewald J, Kupfer D. Functional neuroimaging evidence for hyperarousal in insomnia. Am J Psychiatr 2004; 161(11): 2126-9.

[28] Levine B, Roehrs T, Stepanski E, Zorick F, Roth T. Fragmenting sleep diminishes its recuperative value. Sleep 1987; 10(6): 590-9.

[29] Mahowald MW, Mahowald ML, Bundlie SR, Ytterberg SR. Sleep fragmentation in rheumatoid arthritis. Arthr and Rheum 1989; 32: 974-83.

[30] Moldofsky H. The contribution of sleep-wake physiology to fibromyalgia. Adv Pain Res and Ther 1990; 17: 227-40.

[31] Currie S, Wilson K, Pontefract A, deLaplante L. Cognitivebehavioural treatment of insomnia secondary to chronic pain. J Cons Clin Psychol 2000; 68(3): 407-16.

[32] Hansen Z, Daykin A, Lamb S. A cognitive-behavioural programme for the management of low back pain in primary care: A description and justification of the intervention used in the back skills training trial. Physiother 2010; 96(2): 87-94.

[33] Morin C, Blais F, Savard J. Are changes in beliefs and attitudes about sleep related to sleep improvements in the treatment of insomnia? Behav Res Ther 2002; 40(7): 741-52.

[34] Jensen I, Bergström G, Ljungquist T, Bodin L. A 3-year follow-up of a multidisciplinary rehabilitation programme for back and neck pain. Pain 2005 Jun; 115(3): 273-83.

[35] Heapy A, Stroud M, Higgins D, Sellinger J. Tailoring cognitive-behavioral therapy for chronic pain: A case example. Journal of Clinical Psychology 2006; 62(11): 1345-54.

[36] Jensen M, Nielson W, Kerns R. Toward the development of a motivational model of pain self-management. The Journal of Pain 2003 Nov; 4(9): 477-92.

[37] Linton S. Understanding Pain for Better Clinical Practice: A Psychological Perspective. Edinburgh: Elsevier, 2005.

[38] Vlaeyen J, Morley S. Cognitive-Behavioral Treatments for chronic pain: what works for whom? Clinical Journal of 
Pain Special Topic Series: Cognitive-Behavioral Treatment for Chronic Pain 2005 Jan; 21(1): 1-8.

[39] Edinger JD, Wohlgemuth WK, Krystal AD, RJ. Behavioral insomnia therapy for fibromyalgia patients: A randomized clinical trial. Arch Intern Med 2005 Nov 28; 165(21): 2527-35.

[40] Morin C, Bootzin R, Buysse D, Edinger J, Espie C, Lichstein K. Psychological and behavioral treatment of insomnia: update of the recent evidence (1998-2004). Sleep 2006; 29(11): 1398-414.

[41] Rybarczyk B, Stepanski E, Fogg L, Lopez M, Barry P, Davis A. A Placebo-Controlled Test of Cognitive-Behavioral Therapy for Comorbid Insomnia in Older Adults. Journal of Consulting and Clinical Psychology 2005 Dec; 73(6): 1164-74.

[42] Morin C. Insomnia: psychological assessment and management. New York: The Guildford Press, 1993.

[43] Perlis M, Jungquist C, Smith MT, Posner D. Cognitive Behavioural Treatment of Insomnia: A Session by Session Guide. New York: Springer, 2005.

[44] Bastien C, Vallières A, Morin C. Validation of the Insomnia Severity Index as an outcome measure for insomnia research. Sleep Medicine 2001; 2: 297-307.

[45] Buysse D, Reynolds C, Monk T, Berman S, Kupfer D. The Pittsburgh Sleep Quality Index: A new instrument for psychiatric practice and research. Psychiatr Res 1989; 28: 193-213.

[46] Blais F, Gendron L, Mimeault V. Évaluation de l'insomnie: validation de trois questionnaires. Encephale 1997; 23: 44753.

[47] Morin C. Dysfunctional beliefs and attitudes about sleep: preliminary scale development and description. The Behavior Therapist 1994; 17(7): 163-4.

[48] Espie C, Inglis S, Harvey L, Tessier S. Insomniacs' attributions, psychometric properties of the Dysfunctional Beliefs and Attitudes about Sleep Scale and the Sleep Disturbance Questionnaire. Psychosomatic Research 2000; 48(2): 141-8.

[49] Cheyne A, Girard T. Spatial characteristics of hallucinations associated with sleep paralysis. Cognitive Neuropsychiatry 2004; 9(4): 281-300.

[50] Takeuchi T, Miyasita A, Sasaki Y, Fukuda K. Isolated sleep paralysis elicited by sleep interruptions. Sleep 1992; 15: 21725.
[51] Argyropoulos S, Wilson S. Sleep disturbances in depression and the effects of antidepressants. International Review of Psychiatry 2005; 17(4): 237-45.

[52] Portenoy R, Ugarte C, Fuller I, Haas G. Population-based survey of pain in the united states: Differences among white, african american, and hispanic subjects. The Journal of Pain 2004 Aug; 5(6): 317-28.

[53] Borbely A. Sleep homeostasis and models of sleep regulation. In: Kryger MH, Roth T, Dement WC, editors. Principles and Practice of Sleep Medicine. 2 ed. Philadelphia: W.B. Saunders; 1994. p. 309-20.

[54] Lavie P. To nap, perchance to sleep - ultradian aspects of napping. In: Dinges D, Broughton R, editors. Sleep and Alertness: Chronobiological, Behavioural and Medical Aspects of Napping.New York: Raven Press; 1989, p. 99-120.

[55] Morin C, Kowatch RA, Wade JB. Behavioral management of sleep disturbances secondary to chronic pain. J Behav Ther and Exp Psychiat 1989; 20: 295-302.

[56] Bjørngaard J, Bjerkeset O, Romundstad P, Gunnell D. Sleeping problems and suicide in 75,000 norwegian adults: a 20 year follow-up of the HUNT I study. Sleep 2011; 34(9): 1155-

[57] Ratcliffe G, Enns M, Belik S-L, Sareen J. Chronic pain conditions and suicidal ideation and suicide attempts: An epidemiologic perspective. Clin J Pain 2008; 24: 204-10.

[58] Tang N, Crane C. Suicidality in chronic pain: A review of the prevalence, risk factors and psychological links. Psychological Medicine 2006; 36(05): 575-86.

[59] van Tilburg M, Spence N, Whitehead W, Bangdiwala S, Goldston D. Chronic pain in adolescents is associated with suicidal thoughts and behaviors. The Journal of Pain 2011 Oct; 12(10): 1032-9.

60] Morin C, Stone J, Trinkle D, Mercer J, Remsberg S. Dysfunctional beliefs and attitudes about sleep among older adults with and without insomnia complaints. Psychology and Aging 1993; 8: 463-7.

[61] Jungquist C, O'Brien C, Matteson-Rusby S, Smith M, Pigeon $\mathrm{W}$, Xia Y, et al. The efficacy of cognitive-behavioral therapy for insomnia in patients with chronic pain. Sleep Medicine 2010 Mar; 11(3): 302-9. 